\title{
NASAL GLIAL HETEROTOPIA-A SERIES OF FOUR CASES
}

Ramani. M, Archana. D, Mandakini, Radhika Krishna. O. H, Krishna Reddy. C. H.

1. Professor, Department of Pathology, Niloufer hospital for women and children

2. Post Graduate, Department of Pathology, Niloufer hospital for women and children

3. Professor, Department of Paediatric Surgery, Niloufer hospital for women and children

4. Assistant Professor, Department of Pathology, Niloufer hospital for women and children

5. Post Graduate, Department of Pathology, Niloufer hospital for women and children

\section{CORRESPONDING AUTHOR:}

Dr. Ramani Malleboyina,

Professor of Pathology,

Niloufer Hospital, Red Hills, Lakdikapul,

Hyderabad-500004.

E-mail: drmramani@sify.com

ABSTRACT: Glial heterotopias are rare, benign, congenital, midline, non- teratomatous extra cranial glial tissues. They may masquerade as encephalocoele or dermoid cyst and mostly present in and around the nose. Clinically, these masses are firm and incompressible. Histologically, they are made up of astrocytes and neuroglial cells, embedded in fibrous and vascular connective tissue. Here in, we present 4 cases of nasal glial heterotopias. The first case was an 8 month old boy who presented with broadening of nose since birth. The second case was of 6 months old girl who presented with a soft tissue swelling over the root of the nose. The third case was of a 2 months old boy who presented with a soft tissue swelling over the nasolabial fold. The fourth case was a 5 month old boy with mass in left nostril. The radiological and histological features along with differential diagnosis are discussed. These cases are presented because of their rarity.

KEY WORDS: Encephalocoele, Glial heterotopias, Non-teratomatous.

INTRODUCTION: Glial heterotopia is a congenital (often midline) developmental, non-neoplastic displacement of neuroglial tissue in extracranial sites without connection to the cranial cavity. It occurs in approximately 1 in 4000 births. There is no gender predilection. Generally presents at birth or within the first few years of life, but may affect any age group. There is no evidence of familial predisposition'.

Most commonly they occur in and around the nasal cavity, where they are traditionally and erroneously termed as 'nasal glioma'. Sixty percent of gliomas are extranasal, 30\% are intranasal, and $10 \%$ are both. Although benign, they can cause significant local damage and cosmetic deformity by compressing and destroying the nasal cartilage and orbital wall ${ }^{2}$. Less commonly, glial heterotopias have been reported in the scalp, tongue, pharynx, palate, orbit, middle ear and neck ${ }^{3}$. Surgery is the treatment of choice and results in complete cure with excellent prognosis. 10\% recurrence or persistence is seen following incomplete removal.

\section{CASE REPORTS:}


CASE 1: An 8 month old boy presented with broadening of nose since birth. There was no H/o difficulty in breathing or bleeding nose. Clinically it was diagnosed as haemangioma of left nostril. CECT of PNS and brain showed mass in left nasal cavity s/o Nasal haemangioma with normal study of the brain.

We received a single grey white to grey brown soft tissue mass measuring $2 \times 1 \mathrm{~cm}$. Cut section showed uniform grey white areas. Microscopy revealed neuroglial tissue with astrocytes and few reactive multinuclear astrocytes admixed in a fibrous and vascular connective tissue. Diagnosis of glial heterotopia in the nasal cavity was made.

CASE 2: A 6 months old girl presented with swelling over root of nose .Clinically diagnosed as nasal dermoid or hamartoma. X-ray revealed a soft tissue swelling over the root of the nose with no calcification. Underlying bone appeared normal.

We received skin covered globular soft tissue mass measuring $2 \times 2 \times 1.5 \mathrm{~cm}$. On cut section it was homogenous and grey white. Histopathology revealed mature glial tissue with multinucleate glial cells in the dermis suggesting a diagnosis of glial heterotopia.

CASE 3: A 2 month old boy presented with swelling in the nasolabial fold since birth, clinically diagnosed as nasolabial haemangioma. CT scan of the skull showed soft tissue swelling in the nasolabial fold with no connection to the cranial cavity.

We received 2 skin covered soft tissue bits measuring $4 \times 1.5 \mathrm{~cm}$ each and other 2 grey white soft tissue bits largest measuring 3x1 cm. Microscopy revealed mature glial tissue with astrocytes arranged in lobules in the dermis suggestive of a glial heterotopia[Figure 2]. Immunohistochemical marker [IHC] Glial Fibrillary Acidic Protein [GFAP] confirmed the diagnosis[Figure 3].

CASE 4: A 5 month old boy presented with mass in left nostril. There was no H/o difficulty in breathing or bleeding nose. Clinically it was diagnosed as haemangioma of left nostril.

We received a single globular soft tissue mass measuring $2 \mathrm{~cm}$ in diameter. Cut section showed uniform grey white areas. Microscopy revealed neuroglial fibers and astrocytes in a background of fibrous and vascular connective tissue. Few multinucleated and gemistocytic astrocytes were also seen. Diagnosis of glial heterotopia in the nasal cavity was made.

DISSCUSION: Heterotopic neuroglial tissue also known as Heterotopic Central Nervous System Tissue (HCNST) or Glial heterotopias or Gliomas, is defined as a mass composed of mature brain tissue isolated from the cranial cavity or spinal $\mathrm{canal}^{3}$. No syndromic predisposition or aetiological factors have been identified in these patients although patients may have other craniofacial anomalies - including cleft palate, micrognathia, and choanal atresia. Our cases had no such associated anomalies.

Since the initial description of heterotopic glial tissue over the dorsal surface of the cervical spinal cord in 1907 by Wolbach, heterotopic brain or glial tissue have been reported in various sites. Most of them were intracranial, leptomeningeal or extracranial midline lesions, and they were considered to represent displaced brain tissue during development ${ }^{4}$. Two terms, heterotopia and encephalocele (encephalic herniation), are often confused. However, they are different and have to be differentially diagnosed because the patients with encephalocele have the potential risk for 
meningitis. The distinction is whether or not there is a patent connection with the cranial cavity, but it is sometimes difficult or even impossible to definitively determine whether a case is heterotopia or encephalocele. Pathogenetically, the commonly accepted theory is that heterotopic neural tissue is a variant of encephalocele ${ }^{5}$.

Glial heterotopias (Gliomas) are locally aggressive lesions usually presenting at birth and $90 \%$ of cases are diagnosed by age of 2 years. Of these, $60 \%$ of gliomas are extra nasal, $30 \%$ intranasal and $10 \%$ of cases are in both sites, communication of the intra and extra nasal components being through a defect in the nasal bone $(2,6)$. They may occur at other sites, such as the paranasal sinuses, nasopharynx, tongue, palate, tonsils and orbit and may be referred to as facial glioma ${ }^{6}$. The diagnosis requires radiographic imaging examinations. The differential diagnosis includes developmental anomalies, inflammatory lesions, traumatic deformity, benign and malignant tumors. After a complex assessment, the management of nasal glial heterotopia is complete surgical excision which offers a cure in most cases.

Extranasal glioma presents as a firm, noncompressible subcutaneous mass, most often located at the nasal dorsum. The mass does not increase in size with crying and does not transilluminate ${ }^{(2,6)}$. The intranasal glioma may present with nasal obstruction, epistaxis or nasal deformity and usually arise from the lateral nasal wall (on examination an intranasal encephalocele arises medially). A clinical sign is the absence of expansion or pulsation of the mass following the compression of ipsilateral internal jugular vein (negative Furstenberg test). The distinction between glioma and encephalocele is very important because of the risk of CSF leak and meningitis 6 . The intranasal lesions are approached by lateral rhinotomy or by endoscopic techniques. The extranasal glioma without an intracranial connection may be excised using either a vertical elliptic midline incision or a horizontal incision over the nasal dorsum.

The diagnosis of these lesions is confirmed by histopathological exams. The lesion is nonencapsulated, composed of large and small islands of glial cells, in a connective fibrous tissue. The glial tissue can be confirmed by immunoreactivity for glial fibrillary acidic protein (GFAP) or S100 protein. Neurons are rare or absent.

CONCLUSION: The nasal glioma is a congenital CNS malformation in which there is anterior displacement of cerebral tissue that has lost their intracranial connection. This must be distinguished from the encephalocele in order to prevent the risk of cerebrospinal fluid (CSF) leak and meningitis. The diagnosis of nervous congenital midline nasal masses requires a complex assessment which must include CT. The treatment of these lesions is surgical excision 6 .

\section{REFERENCES:}

1. Bruce M, Weing. Non-Neoplastic lesions of the Nasal cavity and Paranasal sinuses. In: Bruce M.wenig, MD. Atlas of Head and Neck Pathology, $2^{\text {nd }}$ Edition.20-22

2. Sitaula et al. Glial Heterotopia of the orbit: A rare presentation. BMC Ophthalmology 2011; $11: 34$.

3. Ay Al-Ammar, H S Al Noumas, M Alqahtani. A midline nasopharyngeal heterotopic neuroglial tissue. The Journal of Laryngology \& Otology 2006; e25, 1 of 4.

4. Jong Im Lee, Ki Kwon Kim, Yoon Keun Park, Kyung Yoon Eah, Jung Ran Kim. Glial Choristoma in the Middle Ear and Mastoid Bone: A Case Report. J Korean Med Sci. 2004; 19:155-8. 
5. Su jin kim, Dae-cheol kim. Glial Choristoma of middle ear-A case report. The Korean Journal of pathology 2007;41:362-5.

6. D. Rusu, S.G. Aprodu, I. Straticiuc-Ciongradi, V. Munteanu, D. Mihaila, P. Plamadeala. Nasal Glial Heterotopia. Journal of Pediatric Surgical Specialties 2008;2:55-7.

7. KH Ma, K-Cheung. Nasal Glioma. Hong Kong Med J 2006; 12:477-9.

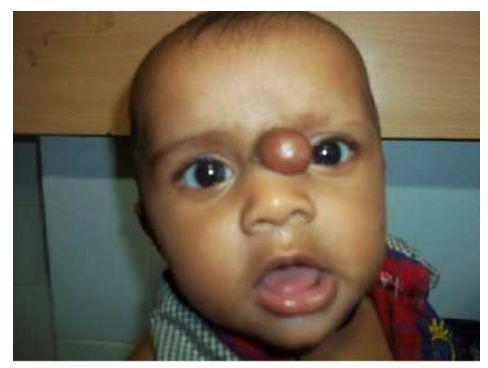

Figure 1: Clinical photograph showing well defined mass at the root of nose.

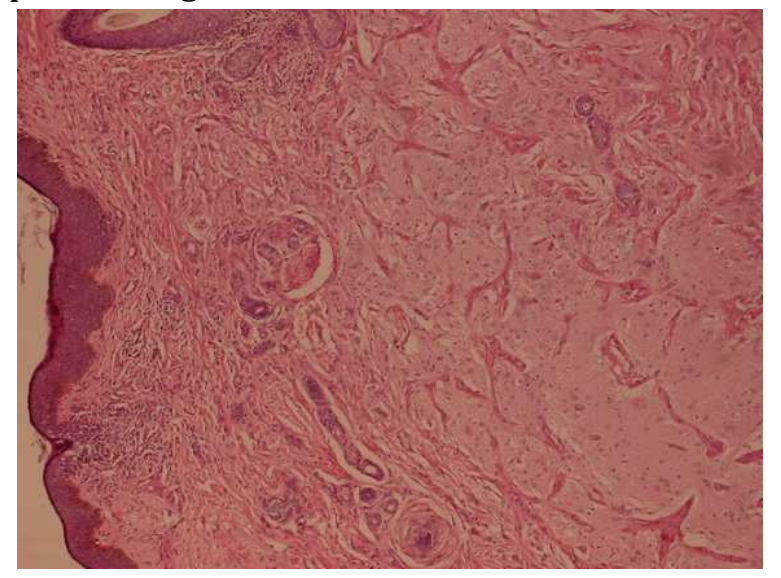

Figure 2: H\&E section showing mature glial tissue with astrocytes arranged in lobules in the dermis.

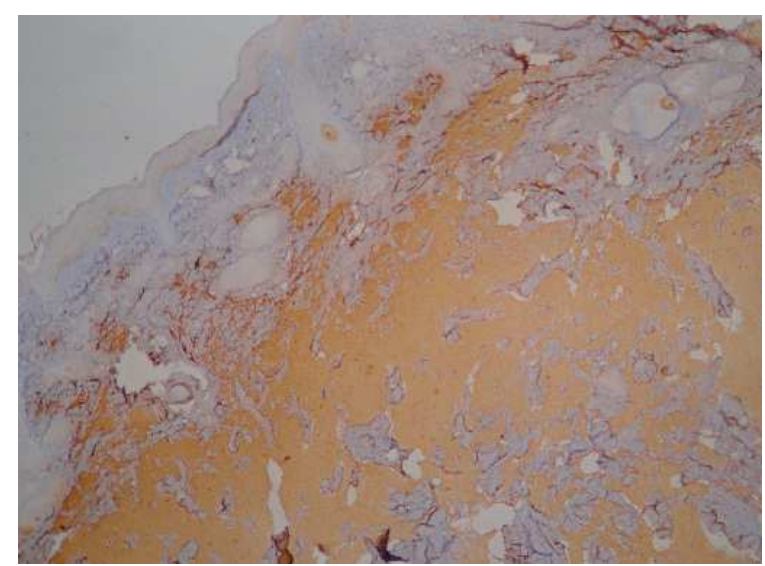

Figure 3: Section showing positivity for glial fibrillary acid protein (GFAP). 